\title{
New antioxidant SkQ1 is an effective protector of rat eye retinal pigment epithelium and choroid under conditions of long-term organotypic cultivation
}

\author{
E. N. Grigoryan ${ }^{1,2^{*}}$, Y. P. Novikova ${ }^{1,2}$, O. S. Gancharova ${ }^{2,3}$, O. V. Kilina ${ }^{2,3}$, P. P. Philippov ${ }^{2,3}$ \\ ${ }^{1}$ Kol'tsov Institute of Developmental Biology, Russian Academy of Sciences, Moscow, Russia; \\ *Corresponding Author: e.grigoryan@hotmail.com \\ ${ }^{2}$ Institute of Mitoengineering, Lomonosov Moscow State University, Moscow, Russia \\ ${ }^{3}$ Belozersky Institute of Physico-Chemical Biology, Lomonosov Moscow State University, Moscow, Russia
}

Received 28 June 2012; revised 26 July 2012; accepted 3 August 2012

\begin{abstract}
Cells have intrinsic mechanisms for cleaning harmful oxidants represented mainly by reactive oxygen species (ROS). Despite the antioxidant defense, ROS can cause serious damage to the retina that with age leads to various eye diseases and even blindness. Among numerous cell sites of ROS generation, mitochondrial electron transport is of crucial importance. Recently, for the purpose of cleaning ROS in the mitochondrial matrix, powerful mitochondria- targeted antioxidant "SkQ1" has been invented. We studied SkQ1 effects upon tissues of rat posterior eye cup that consisted: retinal pigment epithelium (RPE) $\leftrightarrow$ choroidal coat $\leftrightarrow$ scleral coat. The eye cups were isolated from the eyes of adult albino rats and cultivated in rotary tissue culture system in the presence of $20 \mathrm{nM}$ SkQ1 or without this compound. After 7 days - 1 month in vitro eye cup samples were studied by immunohistochemistry, routine histology, morphometry, and digital image analysis. We have found that under chosen, "in vitro like in vivo" conditions $20 \mathrm{nM}$ SkQ1 effectively reduced cell death in RPE and choroid, protected RPE from disintegration caused by cell phenotypic transformation and withdrawal from the layer, suppressed transmigration of choroidal coat cells. In the ex vivo model we used degenerative processes were more pronounced in the eye cup center where SkQ1 effect was most vivid. All this give us hopes for effectiveness of SkQ1 treatment of retinal central part that is very susceptible to light-induced over-oxidation injury and mostly suffering in many age-related diseases, AMD, in particular.
\end{abstract}

Keywords: Rat; Eye; Retinal Pigment Epithelium; Choroid; Organotypic Culturing in Vitro; AMD Remodeling; Antioxidant SkQ1; Cell Behavior; Cell Death

\section{INTRODUCTION}

Cells possess an array of antioxidant mechanisms to clean harmful oxidants represented mainly by reactive oxygen species (ROS). These mechanisms include hydrophilic radical scavengers such as ascorbate and glutathion, lipophilic scavengers such as carotenoids, tocopherols, flavonoids and ubiquinol, a number of various enzymes converting ROS into less dangerous or not dangerous compounds. Despite of the protective role of natural antioxidants, ROS can cause a serious damage to cells. This is especially true for the retinal cells, mainly for photoreceptor and RPE cells, which are highly susceptible to oxidative stress because of a very high rate of oxygen metabolism and concentration of polyunsaturated fatty acids in photoreceptor membranes. These features of retinal cells together with lifelong exposure to light are auspicious conditions for permanent production of ROS within the retina. Repetitive oxidative stress triggered by ROS leads to damage of the outer neural retina, retinal pigment epithelium (RPE), Bruch membrane and choroidal coat that, in turn, initiate a development of various eye diseases [1-6]. ROS are accumulating during time (age) and because of this some of eye diseases are strongly age-related and develop in patients of elderly age sinking their life quality.

Among numerous sites of ROS generation in the cell, mitochondrial electron transport is of crucial importance [7-9]. To clean the matrix space of mitochondria, the place of ROS accumulation in the cell [8], a novel class of antioxidants has recently been invented [8-14]. The history of these antioxidants started when V. Skulachev $[15,17]$ suggested synthetic hydrophobic ions named later "Sku- 
lachev (Sk) ions" [16]. In the ions in question, the charge of the ionized atom is distributed over a rather large molecule and, as a result, the electric forces attracting water dipoles to the ion are too weak to arrange a water layer around the ion. That is why the cations of such a type penetrate into energized mitochondria easily, so that the final ratio of concentrations of the cations inside and outside the matrix can be a value of several orders of magnitude. It means that even an uncharged hydrophobic compound conjugated with the penetrating cation should be accumulated effectively in the matrix of energized mitochondria [17]. Due to this consideration, the penetrating cations have been suggested for use as "electric locomotives" for the accumulation of uncharged compounds inside mitochondria [15,18-19]. This approach has been used also by Murfy's group to develop a penetrating antioxidant, mitoQ, which represents ubiquinone covalently attached by an aliphatic 10-carbon chain to a lipophilic triphenylphosphonium cation [10].

Quite recently, Skulachev V.P. and colleagues synthesized a novel penetrating cation 10-(6'-plastoquinonyl) decyltriphenylphosphonium [11] composed of plastoquinone conjugated with phosphonium. The cation designated SkQ1 appeared to be a powerful antioxidant in a number of in vitro and in vivo models [11-14]. Here we describe for the first time SkQ1 effect upon cell viability, differentiation and migration in the complex RPE $\leftrightarrow$ choroid $\leftrightarrow$ sclera isolated from the eye of adult albino rat. Results we obtained show that in chosen conditions of organotypic long-term culturing of the rat posterior eye cups $20 \mathrm{nM}$ SkQ1 added to the culture medium significantly conserves the intact state of the tissues: reduces cell death, prevents RPE from cell withdrawal and phenotype change, and, finally, suppresses transmigration of endothelial cells of choroid through Bruch's membrane and RPE.

\section{MATERIALS AND METHODS}

Compound SkQ1 was synthesized as described in [11]. Our preliminary tests of SkQ1 effect upon rat eye tissues revealed that the concentration of tens of $\mathrm{nM}$ is the most effective as compared with that of units or hundreds nM. That is why we then used SkQ1 in $\mathrm{nM}$ concentrations and $20 \mathrm{nM}$ SkQ1 in this particular work.

Samples of eye cups were obtained from the eyes of narcotized adult ( 2 - 3 months) albino rats Wistar. Maintenance, anaesthesia and utilization of the animals were carried out in accordance with bioethics regulations of Russian Academy of Sciences. The procedures of eye cups obtaining, rotary cultivation, samples' fixation as well as the methods of the study of eye cup tissues are described in detail in [20]. Briefly, the eye anterior segment, containing the cornea, iris, and lens, was removed by means of microsurgery. Then the eye posterior segments were in- cubated for 3 - $5 \mathrm{~min}$ in $5 \mathrm{ml}$ commercial medium DMEM with phenol indicator (Dulbecco's Modified Eagle's Medium, Invitrogen), containing $3 \mathrm{mg}$ L-glutamine (Helicon, Moscow), 4\% gentamicin (LEK Pharmaceuticals, Moscow) (medium A) and 10 mM EDTA. Afterward, the neural retinas were removed and samples of posterior eye cups put into flasks, containing medium A, 10\% fetal calf serum (Rusbiolink), and $20 \mathrm{nM}$ SkQ1 synthesized at the Institute of Mitoengineering (MSU). In the control SkQ1 was not added to the medium. Then the flasks (6-SkQ1 containing, and 6-control, without SkQ1, in each from three tests) were placed into the roller (RM-1, Elmi, Latvia) and cultivated in a dark sterile box at $60 \mathrm{rpm}$ and $35.5^{\circ} \mathrm{C}$ for 7,14 and 30 days. The culture medium was not replaced during the time period of rotation. After culturing, the samples were fixed in Bouin's solution or $4 \%$ formaldehyde.

Morphological, quantitative morphometric, and digital image analyses of RPE and choroid. Samples of eye posterior segment fixed in Bouin's solution were investigated by methods of routine histology. Serial, paraffin 7 $\mu \mathrm{m}$ cross-sections were obtained and then stained with hematoxylin-eosin. Then they were analyzed using Jenaval (Carl Zeiss, Jena) and Olimpus AH-3 microscopes. Images obtained were registered by digital camera and computer (software Lite) and studied using visual evaluation and Corel Draw, Adobe Photoshop, Excel and Plot Calc computer programs.

To estimate the viability of cells, an average number of pyknotic nuclei we counted relative to the total number of nuclei in the RPE layer on 100 - 150 cross-sections of the periphery and central region of each eye cup sample. The frequency of the phenotype transformation of the RPE cells was determined in the same cross-sections by counting the withdrawn cells in proximity of the RPE layer, which also lost epithelial morphology; rare mitotic cells were also counted. In addition images obtained were registered by digital camera and computer (software Lite) and studied using visual evaluation and computer programs. Eye cup samples obtained on 14 day of in vitro culturing we used for estimation of a relative extension of destroyed regions of RPE layer. For that we made digital photos of the serial slices of cultivated eye posterior cups and then taken images were treated by means of Corel Draw, Adobe Photoshop, Excel and Plot Calc. Statistic analysis of the data was carried out using Microsoft Excel 2010. That made possible to evaluate the dimensions of RPE layer and those of regions of RPE destruction and bring the data in correlation with each other.

Immunochemical analysis of RPE layer in the content of cultured posterior eye cups. Samples of eye cups were fixed in $4 \%$ formalin, frozen in liquid nitrogen and stored at $-20^{\circ} \mathrm{C}$ before cryocut. The frozen cross-sections $(10 \mu \mathrm{m})$ were dried and then used for treatment over standard pro- 
tocol of immunostaining. To reveal the traits of phagocytic differentiation in RPE cells, Mouse Anti-Human Macrophage antigen Monoclonal Antibody, Unconjugated, Clone LN-5 (Sigma-Aldrich) was used in working concentration 1:100. Protein by biuret was $38 \mathrm{MG} / \mathrm{ML}$ as mentioned in certificate of analysis. We used working concentration 1:100 (1:400-recommended). Therefore the exact concentration of the protein was $0.38 \mathrm{mg}$ in $1 \mathrm{ml}$ of the primary antibody solution.

Secondary antibody-anti-mouse IgG-FITC conjugated (Sigma) - was used in concentration 1:50 (1:64recommended). Protein by UV absorbance $4.2 \mathrm{mg} / \mathrm{ml} \mathrm{men-}$ tioned in certificate of analysis. Therefore the exact concentration of the protein was $0.08 \mathrm{mg}$ in $1 \mathrm{ml}$ of the secondary antibody solution. In our experiment we used pretests (Abs titration) for checking up the specificity and intensity of the fluorescent signal getting after Abs application. Afterwards cross-sections were washed trice and placed into Prolong (Antifade Kit, Molecular Probes) under cover glasses. The cross-sections similarly treated but in the absence of primary antibody served as a control of specificity.

TUNEL (Terminal deoxynucleotidyl Transferase Biotin-dUTP Nick End Labeling) assay was performed after 7 and 14 days of eye cup culturing using the DNA fragmentation assay kit (Promega). A number of TUNELlabeled cells was visually evaluated and calculated on the stained sections and digital photographs made at the equal magnification (ocular $10 \times$, objective $20 \times$ ) and photography regime for SkQ1 treated and control samples.

\section{RESULTS}

Cell morphology, viability, and behavior in the posterior eye cup tissues we studied on the $7^{\text {th }}, 14^{\text {th }}$ and $30^{\text {th }}$ days of rotary culturing in the presence or absence of $20 \mathrm{nM}$ SkQ1.

After 7 days of roller cultivation in the absence of SkQ1 the overall structure and layering of the eye cup complex did not change. However, pictures taken with high magnification showed the dense granular parietal chromatin in some nuclei suggesting cells were entering into apoptosis. A number of those cells averaged 15\% - 20\% of the total RPE population and was higher $(17.6 \pm 4.0$ per section) at the centre (eye cup bottom) than at the periphery $(4.3 \pm 2.0$ per section). Some RPE cells detached from Bruch's membrane and migrated to the vitreal direction (Figure 1A). The withdrawn RPE cells underwent macrophagal transformation and, similar to resident macrophages, demonstrated a specific immunoreaction with antibodies against macrophagal antigen (Figure 2A).

However, the morphology of the transformed RPE cells differed from that of macrophages and served for RPE cell identification. RPE cells leaving the layer contained one, or more often two, large diffusely-stained oval nuclei localized in the center of a cell. Whereas macrophages had one larger tightly-stained crescent nucleus shifted to the cell periphery (not shown). Transformation of RPE cells to the macrophagal phenotype was more pronounced in the centre of the eye cup than in its periphery. Direct counting of the number of transformed RPE cells on the serial cross-sections gave the values of $20.0 \pm 5.8$ and 9.0 \pm 2.3 per section for central part of RPE and its periphery, respectively (Figure 3).

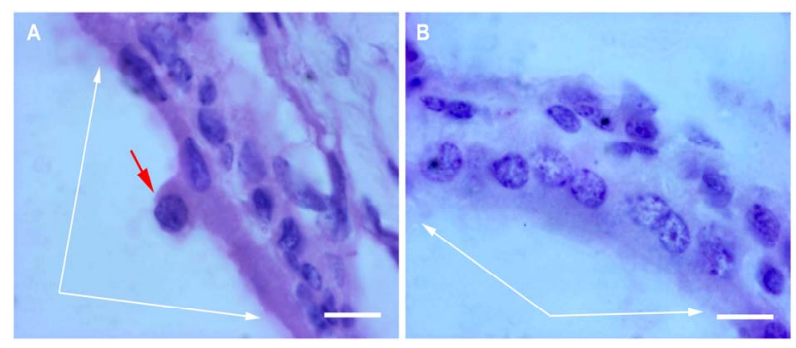

Figure 1. RPE after 7-day organotypic culturing of rat posterior eye cup. $20 \mathrm{nM} \mathrm{SkQ1}$ was absent (A) or present (B) in the culture medium. White arrows indicate the RPE layer; a withdrawn RPE cell (red arrow) can be seen in A. The cross-sections stained with hematoxilin-eosin. Scale bar $10 \mu \mathrm{m}$.
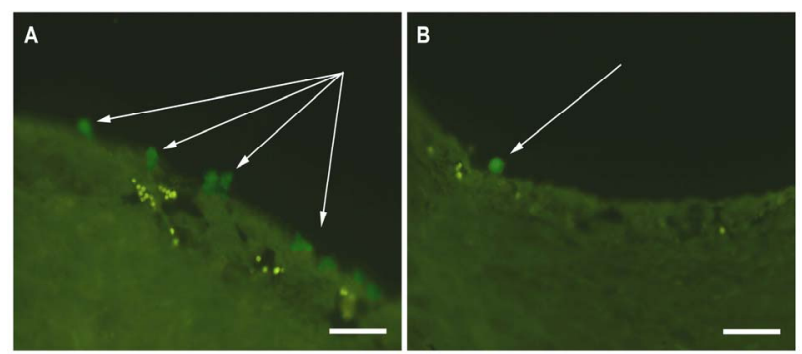

Figure 2. Immunodetection of RPE cells undergone macrophagal phenotypic transformation after 7-day organotypic cultivation of the eye cup. $20 \mathrm{nM}$ SkQ1 was absent (A) or present (B) in the culture medium. Arrows indicate the transformed RPE cells. The complex cross-sections stained with antibody against macrophagal antigen. Scale bar $50 \mu \mathrm{m}$.

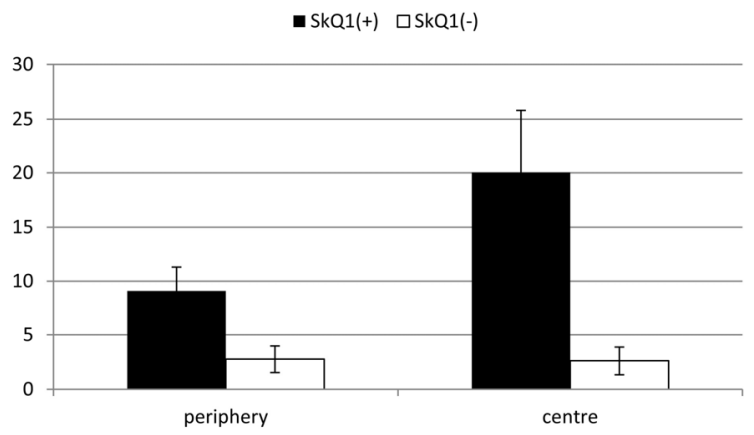

Figure 3. Average number (per one tissue slice) of transformed RPE cells after 7-day organotypic culturing of the eye cup. Calculation has been done separately for the periphery (left) and the center (right) of the eye cup. $20 \mathrm{nM} \mathrm{SkQ1} \mathrm{was} \mathrm{absent}$ (empty bars) or present (filled bars) in the culture medium. The data presented have been obtained after statistical analysis of the data calculated for 100 tissue slices. 
A few mitotic cells, mainly at the eye cup periphery, were found in the RPE layer suggesting realization of RPE cell ability to divide under stressful conditions.

According to TUNEL assay, the number of dead cells in the RPE and choroid layers as a whole was equal to $20 \%-30 \%$ of the total number of cells in these tissues (not shown).

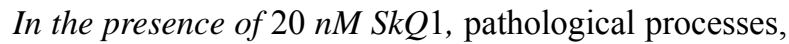
revealed in the RPE and choroid layers at the $7^{\text {th }}$ day of rotary culturing of rat posterior eye cup in the absence of SkQ1, were only feebly noted (Figure 1B) and (Figure 2B). Here, the number of transformed RPE cells was 2.6 \pm 1.3 and $2.8 \pm 1.2$ per section for the centre and periphery of the layer, respectively (see Figure 3). (Compare with the corresponding values of $20.0 \pm 5.8$ and $9.0 \pm 2.3$ per section in the absence of SkQ1). Thus, the protective effect of $20 \mathrm{nM} \mathrm{SkQ1}$ reached $\sim 8$ in the centre and $\sim 2$ in the periphery of the eye cup. In this case, there were no RPE cells in the mitotic phase. Counting of pyknoses gave a number of dead cells in the RPE lightly exceeded $5 \%$ on average of the total cell population in this tissue. Remarkably, the number of dead cells differed in the centre and periphery of the eye cup and comprised $5.1 \pm$ 2.6 and $1.4 \pm 1.4$ per section, respectively.

After 14 days of rotary culturing in the absence of SkQ1, pathological processes in the RPE $\leftrightarrow$ choroids $\leftrightarrow$ sclera became much more evident when compared with 7 days in vitro (Figure 4). Though the tissues of the complex were still conserved, the RPE layer appeared to be partly destroyed because of RPE cells' death and frequent withdrawal from the layer. Binuclear RPE cells which left the layer were present not only at the RPE inner surface but often far from the layer. Clusters of cells in the RPE proximity were often presented by phagocytizing withdrawn RPE cells as well as by the resident macrophages. Besides, extremely large in size, phagocytizing cells were found in RPE layer. They contained absorbed remnants-large brown granules of dying neighboring RPE cells. According to the computer morphometry, the value of RPE layer disintegration, caused by its cell withdrawal, was higher in the central region $(12 \%)$ than in the periphery $(2 \%)$. Another finding registered on the $14^{\text {th }}$ day of culturing in the absence of SkQ1 was an intensive transmigration of choroidal cells via RPE layer and Bruch's membrane in the direction of the eye cup "cavity". As a consequence, the ordered structure of the choroid was disrupted, choroidal and RPE cells got intermixed. This resulted in the "washing out" of the border between the RPE and choroid layers (Figure 5). As in the case of RPE, these events were more pronounced in the centre of the eye cup than in its periphery. Visual analysis (Figure 5D) and TUNEL assay (not shown) revealed a number of pyknotic and mitotic cells in the layer.

In the presence of $20 \mathrm{nM} S k Q 1$, only some of the cells

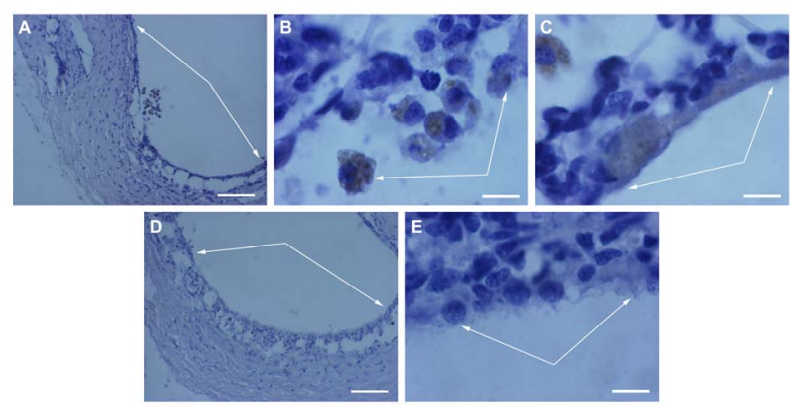

Figure 4. RPE after a 14-day organotypic culturing of the rat posterior eye cup. $20 \mathrm{nM}$ SkQ1 was absent (A, B, C) or present (D, $\mathrm{E})$ in the culture medium. General view (A, D) of the RPE layer (arrows); fagocytizing RPE cells (arrows in B and C) withdrawn from (B) and remained in (C) the RPE layer; a fragment of the RPE layer E. The complex cross-sections stained with hematoxilin-eosin. Scale bar: $100 \mu \mathrm{m}$; (A, D) and $10 \mu \mathrm{m}$ (B, C, E).
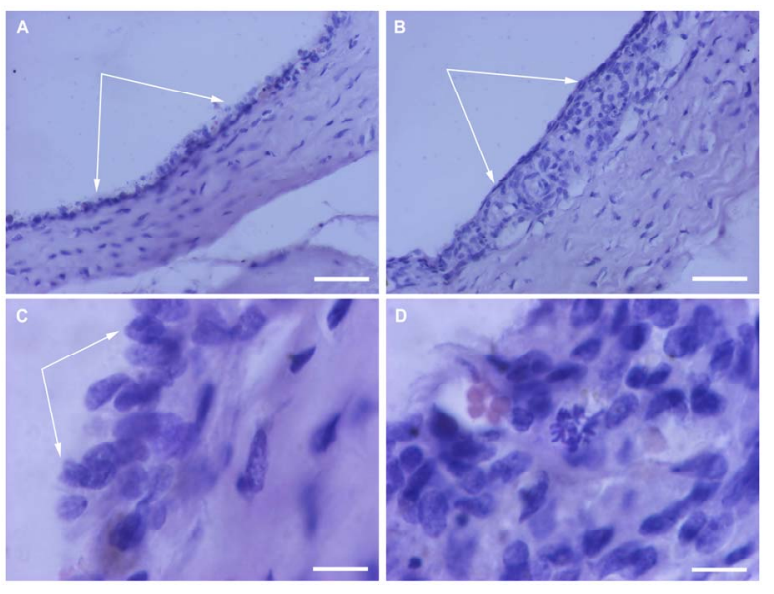

Figure 5. Choroid cells after a 14-day organotypic cultivation of the RPE-choroid-sclera complex. $20 \mathrm{nM}$ SkQ1 was absent $(\mathrm{A}, \mathrm{C}, \mathrm{D})$ or present $(\mathrm{B})$ in the culture medium. General view (A, B) of the choroid coat (arrows). Transmigration of choroid cells $(\mathrm{A}, \mathrm{C})$ and mitosis (D, red arrow) in the choroid are detected in the absence but not in the presence (B) of SkQ1. The complex cross-sections stained with hematoxilin-eosin. Scale bar: $50 \mu \mathrm{m}(\mathrm{A}, \mathrm{B})$ and $10 \mu \mathrm{m}(\mathrm{C}, \mathrm{D})$.

withdrew from RPE layer which still maintained its general integrity with the choroidal and scleral coats (see Figure 4). Cytoplasm of RPE cells contained much less absorbed cell debrises and remnants or did not contain those at all. The morphometrical estimation of RPE disturbance extension in the central part and periphery of the layer showed 2 times less values than those determined in the absence of SkQ1. The thickness of the RPE $\leftrightarrow$ choroid unit and that of the choroid alone was in the main close to initial: a large migration of choroid cells was not mentioned, though a few choroidal cells migrated through RPE layer, merely in particular loci of choroid. The boundary between two layers remained morphologically plane and was not occupied by endothelial cells of the choroid (see Figure 5). The number of pyknotic cells in the 
RPE $\leftrightarrow$ choroids $\leftrightarrow$ sclera was less than that in the absence of SkQ1. That was consistent with the data of TUNEL assay detecting 2-times less dead cells in the presence of SkQ1.

After 30 days of rotary culturing in the absence of SkQ1, morphological analysis of the rat eye posterior cups revealed that only scleral coat kept integrity and its cellsfibroblasts were still alive. Meanwhile the RPE and choroid layers were entirely destroyed, and their cells-dead (Figure 6A).

In the presence of $20 \mathrm{nM} S k Q 1$, the RPE $\leftrightarrow$ choroid complex could detach from the scleral coat but its architecture and, in particular, the integrity of the RPE layer were still preserved (Figure 6B).

\section{DISCUSSION}

V. Skulachev group synthesized an antioxidant attached to a positively charged ion, called SkQ1. This compound passes through the cell membrane and travel to the mitochondrial intermembrane space, where it soaks up any ROS formed by the electron transport chain. Studies by the group also showed that SkQ1 proved beneficial for heart and cardiovascular diseases, tumor growth, and cataracts [11-14]. All mentioned as well as other remodeled disorders represented typical, frequently occurring, agerelated health problems and that, in turn, brings SkQ1 to the front line of the defense from the negative accompaniment of aging.

Vision loss in AMD occurs through photoreceptor damage, abnormalities in the retinal pigment epithelium, Bruch's membrane + choriocapillaris [2]. A number of in vitro and in vivo models have been employed to find the protective effects of various antioxidants upon the retina. In this work, we used an organotypic cultivation of the rat posterior eye cups, consisting of the RPE $\leftrightarrow$ choroid $\leftrightarrow$ sclera complex, in a roller [20], to examine the effect of the new antioxidant SkQ1 [8, 9] upon RPE and choroid. The advantage of this method as compared with any of in vivo models is its relative simplicity and convenience. In comparison with known in vitro models the method, being an ex vivo approach, provides the possibility to study of retinal structures in their complex and
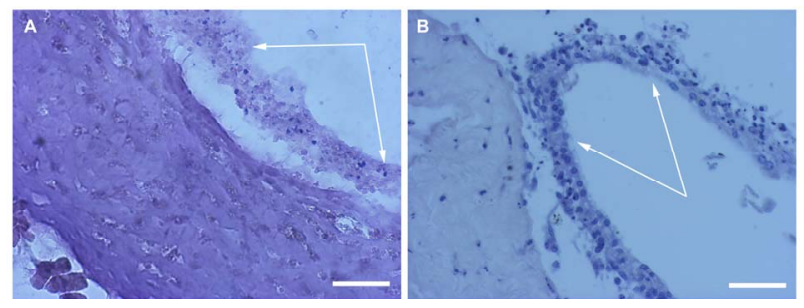

Figure 6. RPE in the content of the eye cup after 30 days of organotypic culturing. $20 \mathrm{nM} \mathrm{SkQ1}$ was absent (A) or present (B) in the culture medium. White arrows indicate the RPE layer. The complex cross-sections stained with hematoxilin-eosin. Scale bar $50 \mu \mathrm{m}$. monitor cell and tissue interactions during a long-term cultivation. That cannot be done using retinal cell lines. During the surgical isolation of the eye posterior cup and subsequent rotary culturing, the tissues are subjected to hypoxia, a deficiency of nutrients, and an attack by fast accumulated ROS. As a result, the cell death and disintegration of the tissues proceed and finally lead to their full disruption. Our data of morphological and immunohistochemical investigation demonstrate that $20 \mathrm{nM} \mathrm{SkQ1}$ once added just before the beginning of rotary culturing suppresses or even prevents destructive processes in posterior eye cup tissues.

In the absence of SkQ1 we have revealed three key pathologic events in the RPE and choroid layers after 7 days of rotary culturing. These were 1) the death of an average of $20 \%$ of the total population of RPE and choroid cells, 2) the detachment of RPE cells from Bruch's membrane and migration of them in the vitreal direction, and 3 ) the acceptance of macrophagal phenotype by RPE cells and withdrawal of them from the layer. Notably, all these events were more pronounced in the centre than in the periphery of the eye cup. After 14 days in vitro noted pathological processes became much more advanced. Thus, gross phagocytizing cells, containing large amount of destroyed neighboring cell remnants, were observed inside RPE layer. In addition binuclear RPE cells filled with cell debris material appeared outside the layer together with true macrophages. In the eye cup samples cultured in the media left of SkQ1 choroidal cells demonstrated transmigration through Bruch's membrane and RPE in the direction of the conditional eye posterior "cavity". This resembled much the processes often accompanying AMD. In addition, a number of pyknotic, mitotic and TUNEL-positive cells registered both in the RPE and choroidal layers. In the course of time all these degenerative processes were more pronounced in the centre than in the periphery of the cultured eye cup. After 30 days in vitro without SkQ1 only the scleral coat was still alive, whereas the RPE and choroid layers underwent structural demolition and cell death.

In contrast in the presence of $20 \mathrm{nM}$ SkQ1, the abovementioned pathological events in the rat posterior eye cup tissues were detected to only a small extent after 7-day rotary culturing. Intact morphology of the majority of RPE cells, low level of apoptosis in the layer, detachment RPE cells from Bruch's membrane, their withdrawal from the layer, rare phenotype transformation as well as choroid cell transmigration - all suggested a good protection of the tissues by SkQ1. Later, in 14 and 30 days in vitro tissues of the rat posterior eye cups kept initial structure, thickness and integration in the complex due to the antioxidative effect of SkQ1. Remarkably, the protective effect of SkQ1 was more evident in the centre than in the periphery of the complex. Therefore, SkQ1 taken in 20 
$\mathrm{nM}$ concentration sustained the viability of RPE and choroidal cells of the retina and prevented the macrophagal transformation of RPE cells under conditions of long-term rotary culturing of the rat eye posterior eye cup.

SkQ1 has been already noted as a very effective antioxidant in various in vitro and in vivo models [11-14]. It is reasonable to suggest that the same is also true in our in vitro model. Among other known antioxidants (glutathione, vitamin C, vitamin E, carotenoids) [2] synthetic SkQ1 in low doses (20 $\mathrm{nM}$ in our case) was appeared to be the effective protector of retinal tissues under stressful in vitro conditions. Be that as it may, the revealed protective effects of SkQ1 in the ex vivo conditions we used allow ones to consider SkQ1 as a potential medication for the treatment of visual disorders, influenced by oxidative and age-related processes in the retina and other eye tissues.

\section{CONCLUSION}

Quite recently, Skulachev V.P. and colleagues synthesized a novel penetrating cation 10-(6'-plastoquinonyl) decyltriphenylphosphonium composed of plastoquinone conjugated with phosphonium. The cation designated SkQ1 appeared to be a powerful antioxidant in a number of in vitro and in vivo models [11-14]. Here we described SkQ1 effect upon tissues of the rat eye posterior cup which being cultivated in vitro demonstrated or remodeled some pathological events specific for age-related macular degeneration in human. In time of 30 day long culturing RPE cells changed their behavior, namely, detached and migrated from the layer, shifted cell phenotype, underwent hyperplasia and apoptosis, which, in turn, all are the hallmark of AMD. Endothelial cells of choroid transmigrated through Bruch's membrane and RPE layer showing the picture closed to that in the wet form of AMD. Therefore mitochondria-targeted antioxidant SkQ1 inserts its vivid effect upon cell viability, differentiation and migration in the complex RPE $\leftrightarrow$ choroid $\leftrightarrow$ sclera of the rat eye keeping all cell and tissue parameters in the limits of the initial norm. Further studies are necessary to proof SkQ1 effect upon neural part of the retina in mammalian eye.

\section{ACKNOWLEDGEMENTS}

We are indebted to Prof. V.P. Skulachev for the useful discussion and to Prof. Keith Rawson-Jones and E.V. Bragina for helping us in the preparation of this article. This work was supported by the grants from the Russian Foundation for Basic Research (09-04-00395-a, 11-0400125-a and ofi-m-2011 11-04-12108).

\section{REFERENCES}

[1] Newell, F.W. (1992) Ophtalmology principles and con- cepts. 7th Edition, Mosby-Year Book, Inc., St. Louis.

[2] Winkler, B.S., Boulton, M.E., Gottsch, J.D. and Sternberg, P. (1999) Oxidative damage and age-related macular degeneration. Molecular Vision, 5, 32.

[3] Emirit, J., Edeas, M. and Brikaire F. (2004) Neurogenerative diseases and oxidative stress. Biomedicine \& Pharmacotherapy, 58, 39-46.

doi:10.1016/j.biopha.2003.11.004

[4] Roth, F., Bindewald, A. and Holz, F.G. (2004) Key pathophysiologic pathways in age-related macular disease. Graefe's Archive for Clinical and Experimental Ophtalmology, 242, 710-716. doi:10.1007/s00417-004-0976-x

[5] Kopitz, J., Holz, F.G., Kaemmerer, E. and Schutt, F. (2004) Lipids and lipid peroxidation products in the pathogenesis of age-related macular degeneration. Biochimie, 86, 825831. doi:10.1016/j.biochi.2004.09.029

[6] Tanito, M., Nishiyama, A., Tanaka, T., Masutani, H., Nakamura, H., Yodoi, J. and Ohira, A. (2002) Change of redox status and modulation by thiol replenishment in retinal photopxidative damage. Investigative Ophthalmology \& Visual Science, 43, 2392-2400.

[7] Beckman, K.B. and Ames, B.N. (1998) The free radical theory of aging matures. Physiological Reviews, 78, 547581.

[8] Skulachev, V.P. (2005) How to clean the dirtiest place in the cell: Cationic antioxidants as intramitochondrial ROS scavengers. Life, 57, 305-310.

[9] Skulachev, V.P. (2006) Bioenergetic aspects of apoptosis, necrosis and mitoptosis. Apoptosis, 11, 473-485. doi:10.1007/s10495-006-5881-9

[10] Kelso, G.F., Porteous, C.M., Coulter, C.V. Hughes, G., Porteous, W.K., Ledgerwood, E.C., Smith, R.A. and Murphy, M.P. (2001) Selective targeting of a redox active ubiquinone to mitochondria within cells. Journal of Biological Chemistry, 276, 4588-4596.

doi:10.1074/jbc.M009093200

[11] Antonenko, Y.N., Avetisyan, A.V., et al. (2008) Mitochondria-targeted plastoquinone derivatives as tools to interrupt execution of the aging program. 1. Cationic plastoquinone derivatives: Synthesis and in vitro studies. Biochemistry (Moscow), 73, 1273-1287. doi:10.1134/S0006297908120018

[12] Bakeeva, L.E., Barskov, I.V., Egorov, M.V., Isaev, N.K., Kapelko, V.I., et al. (2008) Mitochondria-targeted plastoquinone derivatives as tools to interrupt execution of the aging program. 2. Treatment of some ROS- and agerelated diseases (heart arrhythmia, heart infarctions, kidney ischemia, and stroke). Biochemistry (Moscow), 73, 1288-1299. doi:10.1134/S000629790812002X

[13] Agapova, L.S., Chernyak, B.V., Domnina, L.V., Dugina, V.B., et al. (2008) Mitochondria-targeted plastoquinone derivatives as tools to interrupt execution of the aging program. 3. Inhibitory effect of SkQ1 on tumor development from p53-deficient cells. Biochemistry (Moscow), 73, 1300-1316. doi:10.1134/S0006297908120031

[14] Neroev, V.V., Archipova, M.M., Bakeeva, L.E., Fursova, A.Zh., Grigorian E.N., et al. (2008) Mitochondria-targeted plastoquinone derivatives as tools to interrupt exe- 
cution of the aging program. 4. Age-related eye disease. SkQ1 returns vision to blind animals. Biochemistry (Moscow), 73, 1317-1328.

[15] Skulachev, V.P. (1998) Membrane Bioenergetics. Springer-Verlag, Berlin.

[16] Green, D.E. (1974) The electromechanochemical model for energy coupling in mitochondria. Biochimica et Biophysica Acta, 346, 27-78. doi:10.1016/0304-4173(74)90011-1

[17] Lieberman, E.A., Topali, V.P., Tsofina, L.M, Jasaitis, A.A. and Skulachev, V.P. (1969) Mechanism of coupling of oxidative phosphorylation and the membrane potential of mitochondria. Nature, 222, 1076-1078.

doi: $10.1038 / 2221076 \mathrm{a} 0$
[18] Severin, S.E., Skulachev, V.P. and Yaguzhinsky, L.S. (1970) A possible role of carnitine in transport of fatty acids through the mitochcondrial membrane. Biochemis$\operatorname{try}($ Moscow), 35, 1250-1257.

[19] Levitsky, D.O. and Skulachev, V.P. (1972). Carnitine: The carrier transporting fatty acids into mitochondria by means of an electrochemical gradient of $\mathrm{H}^{+}$. Biochimica et Biophysica Acta, 275, 33-50. doi:10.1016/0005-2728(72)90022-9

[20] Grigoryan, E.N., Novikova, Yu.P., Kilina, O.V. and Philippov, P.P. (2007). New method of in vitro culturing of pigment retinal epithelium in the structure of the posterior eye sector of adult rat. Cell Technologies in Biology and Medicine, N4, 618-625. 